\title{
A Review of Freely Available, Open-Source Software for the Automated Analysis of the Behavior of Adult Zebrafish
}

\author{
Juan E. Franco-Restrepo,, Diego A. Forero, ${ }^{1,2}$ and Rafael A. Vargas, ${ }^{1,2}$
}

\begin{abstract}
The analysis of behavior in animal models is an important objective in many research fields, including neuroscience, psychology, toxicology, and neuropsychopharmacology. Animal models have been used for many years, and several behavioral paradigms, such as locomotor activity, social interactions, and cognitive behavior, have been studied in animal models to correlate the behaviors with pharmacological or environmental interventions and with molecular, biochemical, and physiological findings. We reviewed the literature looking for open-source, freely available software to analyze animal behavior and found 12 freely available programs: ToxTrack, EthoWatcher, Mouse Behavior Tracker, Mouse Move, JAABA, wrMTrck, AnimalTracker, idTracker, Ctrax, Mousetracker, VideoHacking, and Cowlog, which were developed with different programs, work on different platforms, and have particular types of inputs and outputs and analysis capabilities. We reviewed some examples of their use, tested some of them, and provided several recommendations for the future development of programs for the automated analysis of behavior in animal models. In conclusion, we show freely available software for the automated analysis of behavior in animal models such as adult zebrafish and provide information for researchers and students looking for quick, easy-to-implement, and inexpensive behavior analysis alternatives.
\end{abstract}

Keywords: animal behavior, neurobiology, computational neuroscience, automated analysis, open-source software, adult zebrafish behavior

\section{Introduction}

$\mathbf{H}$ ISTORICALLY, ANIMAL MODELS have been used for a long time in medical and biological research, having their origins in ancient Greece with Aristotle (384-322 BC). ${ }^{1}$ In 1902, Lucien Cuénot used mice (Mus musculus) for the first time to investigate Mendelian inheritance, and in 1903, William Castle used fruit flies (Drosophila melanogaster) to study the heritability of albinism. ${ }^{2}$ In 1822 , zebrafish (Danio rerio) were described by Hamilton, and in the 1980s, the zebrafish emerged as a novel organism for research in many fields to study biological phenomena. ${ }^{3,4}$ These three animal models have complete genome sequences that make them suitable for behavioral studies that correlate with molecular analyses. In comparison with the human genome sequence, genes from mice have $\sim 80 \%$ homology, ${ }^{5}$ zebrafish $70 \%,{ }^{4}$ and fruit flies $60 \% .^{6}$ These genetic similarities allow the study of human diseases and biological phenomena in animal models, in which it is necessary to perform behavioral analyses, among other systematic studies. The behavior description, or ethography, is a task that requires time by a trained researcher to recognize and analyze behavior patterns in animal species. For example, aggressive behavior may be recognized as follows: chasing and biting patterns in mice and adult zebrafish. ${ }^{7,8}$ Traditionally, this type of behavior is recorded, and the results are manually extracted.

Many of the behavioral tests commonly used in several animal models are an adaptation from the scientific literature available in mouse models. The three main types of behavioral domains evaluated in animal models, including adult zebrafish, are emotional behavior (e.g., anxiety or fear), cognitive behavior (e.g., spatial memory, object recognition, or learning), and social interaction (e.g., aggressiveness, social preference, and courtship), as evidenced in several publications. $^{9-12}$ For instance, the analysis of locomotor behavior can be explored in different species and with several

\footnotetext{
${ }^{1}$ Laboratory of Neuropsychiatric Genetics, Biomedical Sciences Research Group, School of Medicine, Universidad Antonio Nariño, Bogotá, Colombia.

${ }^{2} \mathrm{PhD}$ Program in Health Sciences, School of Medicine, Universidad Antonio Nariño, Bogotá, Colombia.
} 
experimental paradigms (e.g., open field in mice, trapezoidal tank in adult zebrafish, and square boxes in flies), and in general, these devices assess the animal's movement in the environment (generating important data, such as total length, velocity, acceleration, and trajectories). On the other hand, there are cognitive tests, such as memory tasks, which assess the number of entries into a region of interest (ROI), time spent in an ROI, and time to reach an ROI. Finally, social interactions can be assessed, and they can have a wide range of presentations; for instance, in larval and adult zebrafish models, it has been found that there are $\sim 190$ evaluable behaviors. ${ }^{8}$ Of these, more than 20 behaviors represent complex actions that are mostly evaluated manually, such as biting, charging, chasing, and fighting (in aggression), changing body color, swimming in circles, following (in courtship), shoaling, and socially interacting. ${ }^{8}$

Although many animal models have been used in neuroscience research to study animal behavior for several decades, only recently has software for the automated analysis of behavior been developed. Software that adapts to the needs of the researcher allows the design of more complex studies, for example, experiments related to studying the influence of the administration of pharmacological substances, genetic modifications, or physical and/or cognitive impairments in animals, testing their specific behaviors in several domains, making assessments in grouped or individual situations, and conducting these analyses with a low rate of error.

This work is focused on reviewing open-source software oriented to study only adult zebrafish behavior and not larval zebrafish behavior. The zebrafish behavior in larval stages has specific characteristics and has been analyzed using software for video and kinematic analysis. ${ }^{13,14}$

Currently, there are several programs for the automated analysis of animal behavior, and although some of these are commercial, an important number of them are open source and free to use. These developments have provided researchers with many advantages, such as saving time and money, improving accuracy, allowing a larger number of animals to be analyzed, and possibly generating highly quantitative and precise results, such as the total length of displacement, velocity, acceleration, turn angles, track trajectories, and number of entries in a ROI. ${ }^{15,16}$

Some commercial programs for the analysis of animal behaviors are EthoVision (Noldus, Inc., Leesburg, VA), ANYmaze (Stoelting Co., Wood Dale, IL), and TopScan (Clever Sys, Inc., VA). These programs require payment for their use. In this article, we focus on freely available open-source software oriented to video analysis, which permits the automated analysis of behavior. It should be noted that some software also needs $\mathrm{C}++$, MATLAB, LabView (National Instruments, TX) commercially available programs, or Python (which needs advanced computational skills) to work.

\section{Literature Review}

For this work, a search was made of the existing literature in PubMed and the academic Google search engine. The keywords for the search included adult zebrafish behavior, animal behavior, automated analysis, and open-source software. Review articles and original articles closely related to the topic were selected and reviewed. Based on this, 12 freely available programs were identified: 3 ImageJ-based pro- grams (wrMTrck, Mouse Behavior Tracker, and AnimalTracker), 2 MATLAB-based image processing programs (idTracker and JAABA), $2 \mathrm{C}++$-based image processing programs (ToxTrack and EthoWatcher), 2 python-based image processing programs (Ctrax and VideoHacking), 1 Pascal/Delphi image processing program (Mousetracker), 1 LabVIEW-based image processing program (Mouse Move), and 1 Java- and HTML-based image processing program (Cowlog). These programs work on different operative systems (Windows, Mac OS, and Linux). In some cases, toolboxes, libraries, and licenses from commercial programming languages (MATLAB, $\mathrm{C}++$, and LabVIEW) are required (Table 1).

\section{Open-Source and Freely Available Software}

The programs mentioned below have in common the semiautomatic analysis of previously recorded videos or the processing of the behavior in real time. Actually, the analysis of behavior through software is performed semiautomatically because the user must adjust the parameters (distance measurements and ROI, mainly), and the software will be responsible for executing the order to follow a specific object. For practical purposes, we will call it automatic analysis. It is important to keep in mind that depending on the software used, the number of steps needed to obtain the behavioral data may be different.

\section{Image processing algorithms}

Processing times may also vary, and it will depend mainly on the video resolution and length, and the software speed. Briefly, the first algorithm commonly used by the software is a segmentation algorithm (i.e., background subtraction followed by threshold definition). It helps to improve animal tracking and to decrease the variations in light in the field (Appendix). After that, animal location (center of mass or animal head) is tracked in $X$ and $Y$ coordinates, and the results are generated. ${ }^{15,16}$ It is also important to note that many algorithms can be customized.

One of the main limitations of the available programs is the difficulty of tracking several animals simultaneously. The main cause for this difficulty is the spatial overlap of animals, which leads to a loss of identity and mixed data. From our point of view, the main advantage and usefulness of tracking multiple individuals are related to the study of locomotor and social behavior, where tracking multiple individuals decreases the amount of time for each analysis. Different techniques, such as the use of visual markers, several cameras, and algorithms based on neural networks, can be used to avoid losing track of the animals; however, it can be invasive, it can be difficult to apply to all animal models, and it increases the complexity of the experiments and the computer memory use. ${ }^{15,16}$

\section{Description of Available Software wrMTrck}

This is a plugin that works with ImageJ. ImageJ is a freely available image processing program developed at the $\mathrm{Na}$ tional Institute of Health (NIH). It was written in the Java programming language and run on Windows, Mac OS, and Linux systems in 32-bit and 64-bit modes. For ImageJ installation, you can visit https://imagej.nih.gov/ij/download.html. 


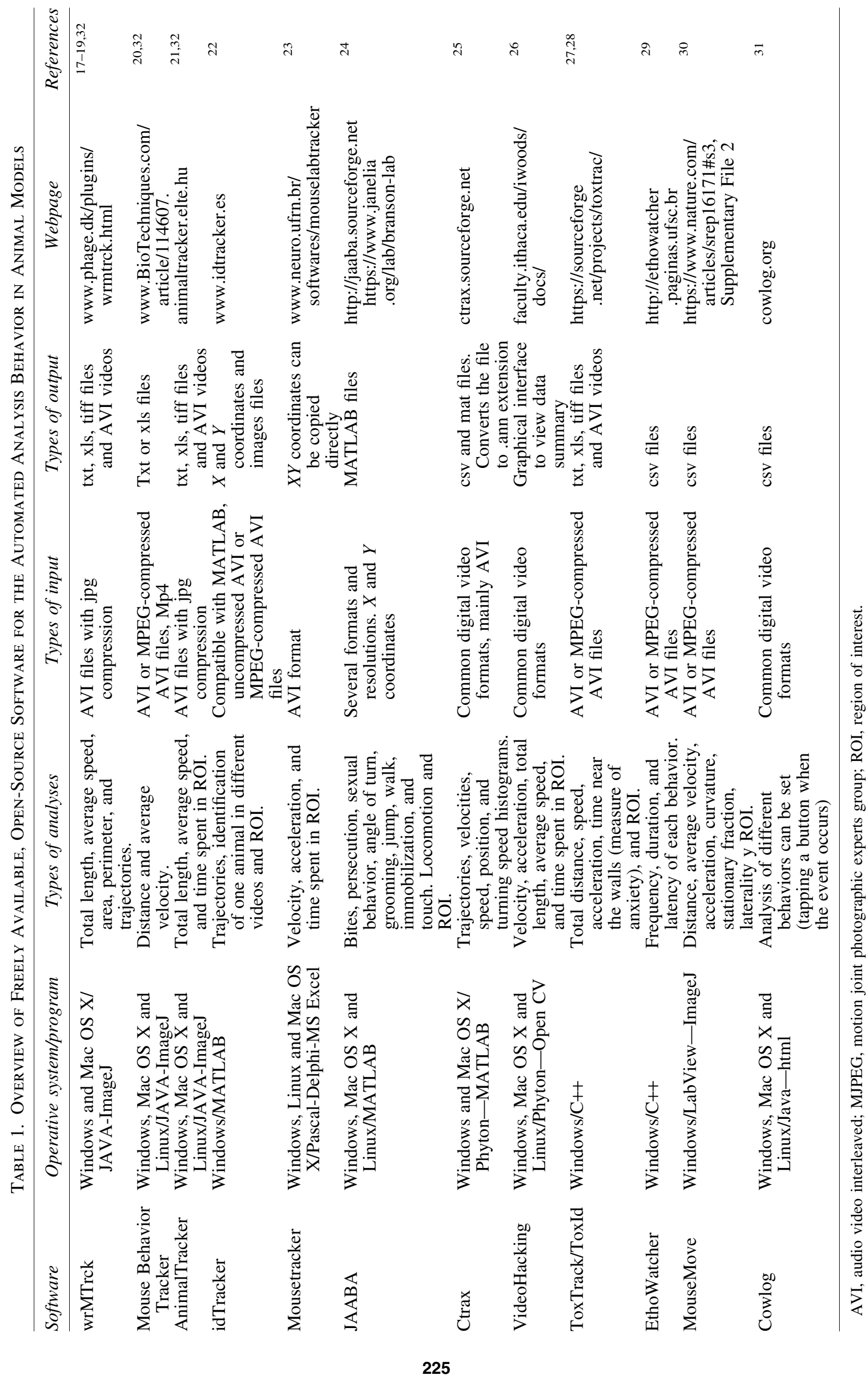


wrMTrck is a plugin created by Jesper Søndergaard Pedersen and his team to study how prion-like proteins spread in Caenorhabditis elegans and to analyze locomotor behavior (www.phage.dk/plugins/wrmtrck.html). ${ }^{17}$ The plugin installation and setup are very easy, and you can read Selvaraj and Santhakumar ${ }^{18}$ for more details. wrMTrck generates raw data, a summary, a video of the tracking analysis, and an image of the path of the animal tracking. Raw data include total distance, number of frames tracked, time points analyzed, maximum speed by frame, tracking area by frames, average speed by frame, and location in $X$ and $Y$ coordinates. The data summary includes the number of objects tracked, total frames analyzed, the number of group of frames tracked, total length, average speed, and area and perimeter measures, among others. Furthermore, processed videos can be saved in Audio Video Interleaved (AVI) format and images in tiff, jpeg, gif, and other common formats. wrMTrck is a useful tool to analyze several behaviors of one animal at a time because it tracks only one animal; the superposition of images from several animals can alter the results when they cross each other, and the data are mixed. Another useful plugin to solve contrast issues is DanioJ 1.0, which increases the illumination threshold for the animal and thus makes the animal more visible for tracking. ${ }^{19}$ It is mainly useful for analyzing locomotor behavior in several species.

\section{Mouse Behavior Tracker}

Mouse Behavior Tracker is a macro developed and implemented in ImageJ and the wrMTrck plugin, which allows automatic tracking of the animal by implementing filters sequentially to maximize the contrast between the animal and the test area (https://www.future-science.com/doi/10.2144/ 000114607/supplementary material). It works in a similar way to AnimalTracker, but with limitations related to obtaining only data for locomotion. This software generates data for distance traveled, speed, and path graphs. In comparison with EthoVision XT (commercial software), there was a correlation of $0.98 \pm 0.03$ (mean \pm SEM, $n=6$ ) in the data. ${ }^{20}$ Mouse Behavior Tracker works in Windows, Mac, and Linux and accepts videos with a resolution of $320 \times 240$ pixels, 30 frames per second (FPS), and Motion Joint Photographic Experts Group (MJPEG) compression, in AVI or MP4 format, with a duration of up to $30 \mathrm{~min}$. It was initially implemented in mice, but can be adapted to any species, allowing the analysis of one individual at a time. This software is useful for the analysis of locomotor behavior exclusively (distance and speed parameters), as mentioned above, and may overestimate data if there are minimal variations of light.

\section{AnimalTracker}

This is a plugin that works with ImageJ, and for installation and more details, you can visit its website (http://animaltracker .elte.hu/). This plugin has three different modules that work together. First, the Tracker module processes the video and facilitates the binarization process to determine the $X Y$ coordinates. The TrackAnalyzer module visualizes the trajectories produced by the Tracker module, and finally, the ZoneDesigner module can be set up to analyze different zones. $^{21}$ AnimalTracker generates data such as total time analyzed, immobility times, distances, and velocity vectors. Furthermore, raw data, means and standard deviations, and highest and lowest values can be identified for distance, time, and immobility times among different ROIs defined in the ZoneDesigner module. The results can be saved as txt or spreadsheet files for further analysis. This software is useful for locomotor behavior and some analysis of social and cognitive behavior. The software is easy to use and requires a few steps; however, it only recognizes one animal at a time, and data mixing can occur when using several animals.

\section{idTracker}

idTracker is a MATLAB package that can be downloaded with MATLAB runtime add-ons from its website (www idtracker.es/). This program can identify several animals in one video, and idTracker has several automated steps that include segmentation to normalize fluctuations of illumination in each frame; fragments of trajectories in which it can identify each fragment of frames when an animal is moving; transformation to generate high contrast; and selection of images that belong to one animal. Images are collected frame by frame, each animal in the video is identified, and these steps are computed and automatically analyzed to give individual trajectories for several animals. Therefore, it is possible to identify a single animal in a group. ${ }^{22}$ One of the main theoretical advantages of idTracker is the possibility to analyze territoriality, leadership hierarchies, and differences between animals in a group solving a task, and identities can be recovered when an animal is out of sight. ${ }^{22}$

\section{Mousetracker}

Mousetracker is an application written in Pascal through a Delphi interface that is able to run on Windows. Microsoft Excel is required to save the results. Up to eight animals can be analyzed at once, and it works through black/white differentiation between the animals and the background, based on a grayscale threshold for animal contrast. ${ }^{23}$ The hardware requirements include a $1.6 \mathrm{GHz}$ processor with $512 \mathrm{MB}$ RAM and a webcam with proper video recording software installed. The program has a simple, intuitive interface with buttons and functions. The videos to be used with this software need low resolution (video setting in AVI format at 4 FPS and $320 \times 240$ pixels). Mousetracker was created at the Brain Institute at the Federal University of Rio Grande do Norte in Brazil and developed to analyze mainly locomotor activity and ROI (the software can be obtained by contact with the software authors at mousetracker@gmail.com).

\section{Janelia Automatic Animal Behavior Annotator}

Janelia Automatic Animal Behavior Annotator (JAABA) is a system that uses machine learning in a similar way to EthoWatcher. It creates a library of learned behaviors by taking a few frames where the event of interest has occurred and the researcher must teach the behavior to the program; it takes 15 to $40 \mathrm{~s}$ to teach each behavior. This software is implemented in MATLAB for Windows, Mac, and Linux operating systems (http://jaaba.sourceforge.net/). ${ }^{24}$ MATLAB runtime add-ons that are freely available for users must be installed to execute the program. JAABA has a large number of algorithms that process characteristics such as position, locomotion, location, wingspan and movement of the wings (tested in flies), and distances between animals, identities, 
and field. The machine learning algorithm requires the graph of the animal's trajectory in $X$ and $Y$ coordinates to find behaviors (learned algorithms) in the video. ${ }^{24}$ Thus, it is necessary to use additional software to create the trajectories. In addition, the user can see the results and identify a behavior that is incorrect. It is applicable to a large number of species and diverse behaviors with an error rate lower than $1 \%$, as tested in flies (adult and larva) and mice. In addition, it was reported that chase behavior analysis by this program had an error rate of $0.6 \%$, while Ctrax showed an error rate of $10 \%$. In the demonstration of its performance, authors processed videos of $1000 \mathrm{~s}(16: 40 \mathrm{~min}){ }^{24}$

From our point of view, it is a very useful tool to evaluate the behavior of multiple animals, offering the possibility of evaluating a large number of social, cognitive, and locomotor behaviors (bites, persecution, sexual behavior, angle of turn, grooming events, jumps, walk, immobilization, touch, etc.) in a large group of animals, saving recording and process ing times in behavioral tests. According to the authors, it is necessary to develop algorithms that encompass other animal species, behaviors, and environments because the JAABA system was focused on flies and mice; however, many behaviors measured by JAABA can be adapted to other species with the same algorithms.

\section{Ctrax}

Ctrax is freely available image analysis software. It was written in Python, and the MATLAB runtime add-ons freely available for users are required. Ctrax can be downloaded online and is widely used. More than 40 publications reported using Ctrax, mainly in D. melanogaster and adult zebrafish (http://ctrax.sourceforge.net/). This software works similarly to others in that it makes a binarization of the animal and then can recognize frame to frame one or several animals (up to 50 flies). ${ }^{25}$ A wide number of analyses can be performed with this software, such as velocity, distance, time spent walking, chase between animals, and a path of several animals in a video. The analysis carried out by the authors included seventeen 30-min trials, each with 20 flies, for a total of 170 flight hours. In addition, similar to JAABA, Ctrax contains a machine learning system that allows the evaluation of additional behaviors, while maintaining the identities of each animal, even over time, with an accuracy of $83.9 \%$ for locomotor behavior and more than $95 \%$ for other behaviors. ${ }^{25}$ This program is the most complete of the software presented in this review. It has the capacity to analyze locomotor behavior (although with less precision than other software) and behavior in the social and cognitive domains as determined by the user.

\section{VideoHacking}

This software works with Python and OpenCV, including the Python libraries matplotlib and NumPy. It consists of several computational modules working together to analyze complex behaviors, such as thigmotaxis, ROI analyses, and, in general, locomotor behavior. The first step is delimiting an ROI in a field, followed by quantification of the locomotor behavior frame by frame, and then allowing the user to group ROIs according to treatment and display the results. Moreover, it contains a graphical interface to view summary data. ${ }^{26}$ Another advantage of VideoHacking is the ability to analyze live or prerecorded videos of several hours with highquality tracking and tracking of several animals simultaneously (as long as they do not overlap). Outcome parameters of locomotor activity, such as velocity, acceleration, total length, average speed, and time spent in an ROI (e.g., thigmotaxis behavior), are generated by the software. Details for its installation are found online, and some computational skills might be needed to solve some problems related to the installation of VideoHacking and OpenCV (https://faculty .ithaca.edu/iwoods/docs/pytracker/). The capacity for the analysis of several hours of testing, the simultaneous testing of multiple animals $(n=48)$, and the adaptation to changes in the lighting conditions offered by this software make it ideal for the analysis of locomotor, social, and cognitive behavior. In addition, the use of this software reduces the presence of the researcher, which reduces variables such as noise levels and lighting changes.

\section{ToxTrack}

ToxTrack is software for Windows, developed in the C++ language (https://sourceforge.net/projects/toxtrac/), which allows the tracking of several animals simultaneously, preserving their identity, even when there are occlusions (loss of continuity of the image). The user can analyze videos recorded previously or in real time (in high resolution, $2048 \times$ 2048 pixels), and the authors recommend that the animals to be followed should be at least 50 pixels in size, the video should be recorded with at least 25 FPS, and no more than 10 20 animals should be tracked in each experiment. ${ }^{27}$ ToxTrack can be used in different animal species, with a tracking efficiency of up to $99.9 \%$. More recently, they developed ToxId (an algorithm implemented in ToxTrack) that identifies animals with 99\% accuracy, even with occlusions and overlapping of animals. This improvement decreases the recognition error rate for an individual from one frame to another and enhances tracking in other species, such as adult zebrafish. ${ }^{27}$ In comparison with idTracker, ToxId ${ }^{28}$ is more efficient in processing videos and decreasing the analysis time, and has an average rate of recognition of several animal models of $97 \%$ compared to $99 \%$ of idTracker. ToxTrack generates results for the total distance, speed, acceleration, time near the walls, and other parameters related to the ROI.

\section{EthoWatcher}

EthoWatcher is a program for Windows, programmed in the $\mathrm{C}++$ language, which allows researchers to make a catalog for the automatic analysis of behavior (http://ethowatcher .paginas.ufsc.br/). It has been used in several species and is useful mainly in rodents. EthoWatcher can evaluate specific behaviors, such as vertical exploration, grooming and sniffing, as well as distance, speed, immobilization, and ROI results (measurement in $\mathrm{cm}$ ), and it generates a plot of paths, using prerecorded or live videos with a length of up to $3 \mathrm{~h}$, with a minimum resolution of $320 \times 240$ pixels, MJPEG compression, and AVI format. ${ }^{29}$ EthoWatcher does not contain preregistered behavior categories; therefore, the initial ethographic analysis is performed by the researcher and then saved as a catalog to be replicated in future analyses. The program reports the frequency, duration, and latency of each behavior and allows the processing of two behaviors simultaneously, which start and end at different times. ${ }^{29}$ The main 
advantage offered by EthoWatcher is the detection of the angles generated at the poles of the animal (head or tail movements) compared to its center of mass, which allows the automatic analysis of the specific behaviors mentioned above. However, it limits the analysis of some behaviors and species.

\section{MouseMove}

This software was developed in LabVIEW 12.0 Development Systems, and it works in the Windows operating system; however, LabVIEW installation is needed to run MouseMove.exe (https://www.ncbi.nlm.nih.gov/pmc/ articles/PMC4632026/supplementary file 2). Furthermore, it is necessary to execute several steps: (1) create a folder with the videos to be analyzed, including an exclusive video of the arena (without animals); (2) open ImageJ and execute the algorithm designed by the authors; (3) identify the folder that contains the videos (ImageJ memory restricts the maximum video length to 5000 frames); (4) the files will be automatically saved in txt format; (5) open MouseMove.exe and open the folder with saved results; (6) it is necessary to reduce the resolution of the video to adjust the ROI in Mouse Move, and any adjustment made in ROI will be processed and saved automatically; and (7) the generated results will be saved in the folder that contains the analyzed videos as csv files. ${ }^{30}$ Mouse Move accepts videos in AVI format with MJPEG compression at 25 FPS and $640 \times 480$ resolution (for optimal performance); however, the macro reduces the resolution to $320 \times 240$ pixels. The variables measured by this software are distance, average velocity, acceleration, curvature, stationary fraction, laterality (number of left and right turns and L/R ratio), and the ability to evaluate novel object recognition using ROI analysis, with a precision greater than $96 \%{ }^{30}$

Mouse Move can be used to analyze the behavior of different animal species and basic parameters of a test; moreover, it offers the possibility of analyzing specific areas of the field, which makes it a useful software for the analysis of locomotor behavior and some social and cognitive behaviors. However, it has limitations in the input resolution of the analyzed video and cannot process specific behaviors.

\section{Cowlog}

Cowlog is another freely available image analysis software. It was implemented using Java and html (http:// 188.166.24.93/download/). From all the programs reviewed in this article, Cowlog is the only one that works with a manual analysis; it can be set up for the analysis of up to 24 behaviors. There are three different versions (Cowlog 1, 2 . and 3), and more than 30 publications report using it in several species. Cowlog is useful when complex behaviors are analyzed, for example, when an animal lays down, sits down, eats, or does another specific task. Furthermore, it works with two windows: a coding window for selecting the videos, scoring the behaviors and selecting the output format, and another window to watch the video. ${ }^{31}$ The generated results are saved each time a behavior is scored and a table with two columns is obtained; the first column lists the behaviors scored, and the second lists the time that the particular behavior occurred. Data can be exported to other programs for further analyses. ${ }^{31}$ This software is not for automatic analysis; however, we consider it useful when an- other software package does not allow the possibility of evaluating a specific behavior.

The programs presented were downloaded and tested on a laptop computer Hewlett Packard, HP Pavilion x360 (processor Intel Core i3, 4 GB RAM, Windows 10, 64 bits). Requirements for some programs are processor Intel Core i5, 8 GB RAM, 64 bits (Toxtrack, IdTracker, Ctrax). In cases where the software worked properly, the programs were tested using recorded videos of 2 min assessing the novel tank test in adult zebrafish (Fig. 1A-D, and Table 1).

\section{Example of Adult Zebrafish Behavior Analysis with Freely Available Software ImageJ Based}

As mentioned before, several programs have been used for the automated analysis of behavior in animal models with multiple species. We are interested in ImageJ, a free, public domain, Java-based image processing program developed at the National Institutes of Health (https://imagej.nih.gov/ij/). ImageJ provides extensibility by Java with thousands of plugins and recordable macros that facilitate scientific image analysis. In addition, this tool has been successfully used worldwide by many laboratories. ${ }^{32}$ Zebrafish $(D$. rerio), both larvae and adults, have previously been used to analyze locomotor and anxiety behavior when exposed to ethanol and other substances, such as fluoxetine, morphine, and nicotine. ${ }^{33,34}$ Briefly, we used two male wild-type adult zebrafish housed in tanks of $7 \mathrm{~L}$ at $27^{\circ} \mathrm{C} \pm 1^{\circ} \mathrm{C}$ and acclimated for 2 weeks before any test. Anxiety and aggressive behaviors were evaluated using the novel tank test and the aggressiveness test. For the novel tank test, we employed a trapezoidal tank, and a video was recorded from the lateral view (Figs. 1B and 2A, C). For the aggressiveness test, a rectangular tank with a mirror on one side was used, and a video was recorded from the top (Fig. 2B, D). A commercial camera was used to record videos. Video record settings were AVI format, MJPEG compressed, 30 FPS, resolution of $840 \times$ 420 pixels, and length of $5 \mathrm{~min}$, and the videos were analyzed with freely available software.

For this example, the software used for the behavior analysis was AnimalTracker, and examples of the results of the paths of the fish for each test are shown in Figure $2 \mathrm{C}$ and D. The results for the parameters of total distance traveled, and average speed and time spent in the ROI (bottom in novel tank test and contact zone for aggressiveness test) are shown in Figure 2E-H.

\section{Discussion}

Current techniques for automatic behavior assessment provide obvious advantages over a manual analysis, facilitating the research process in several fields. Several programs presented in this study evaluate a wide range of behaviors, and depending on the species, they can be adjusted for different analyses. When a video analysis is performed, you need to consider how most programs work. Few programs, as expected, work exclusively on the Windows operating system, and most are also available for MacOS and Linux. The main format supported by several programs is AVI with MJPEG compression, and problems can occur in several steps, such as software installation, formatting of the input videos, handling the video illumination, and exporting data; however, among the most important factors mentioned are 

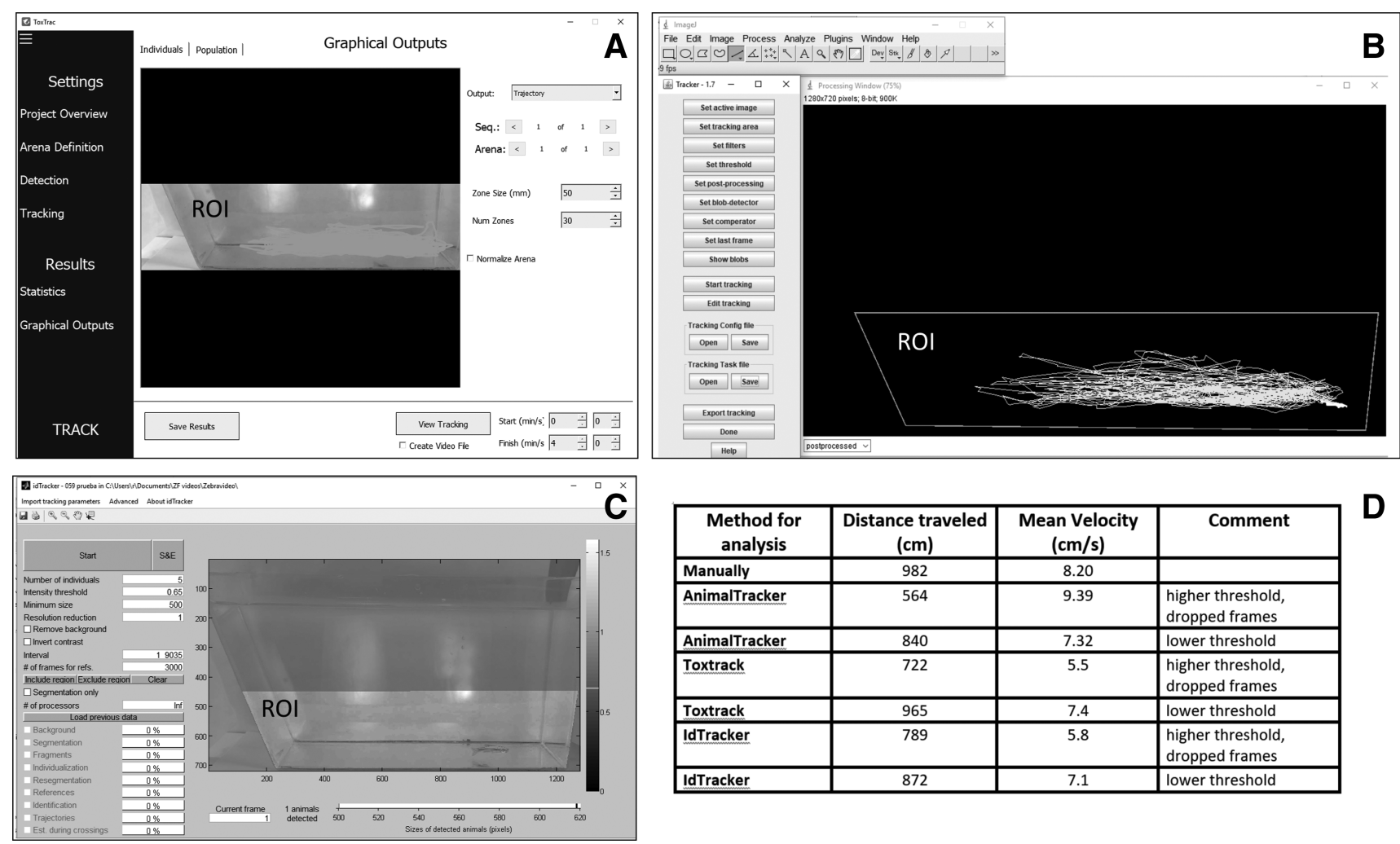

\begin{tabular}{|l|c|c|l|}
\hline $\begin{array}{c}\text { Method for } \\
\text { analysis }\end{array}$ & $\begin{array}{c}\text { Distance traveled } \\
\text { (cm) }\end{array}$ & $\begin{array}{c}\text { Mean Velocity } \\
\text { (cm/s) }\end{array}$ & \multicolumn{1}{|c|}{ Comment } \\
\hline Manually & 982 & 8.20 & \\
\hline AnimalTracker & 564 & 9.39 & $\begin{array}{l}\text { higher threshold, } \\
\text { dropped frames }\end{array}$ \\
\hline AnimalTracker & 840 & 7.32 & lower threshold \\
\hline Toxtrack & 722 & 5.5 & $\begin{array}{l}\text { higher threshold, } \\
\text { dropped frames }\end{array}$ \\
\hline Toxtrack & 965 & 7.4 & lower threshold \\
\hline IdTracker & 789 & 5.8 & $\begin{array}{l}\text { higher threshold, } \\
\text { dropped frames }\end{array}$ \\
\hline IdTracker & 872 & 7.1 & lower threshold \\
\hline
\end{tabular}

FIG. 1. Freely available and open-source video analysis programs. Several programs were evaluated using the same short video ( 2 min, $30 \mathrm{FPS}$, and $320 \times 240$ pixels) obtained in an anxiety test, using a trapezoidal tank, of an adult zebrafish. Two basic measurements, the total length of displacement and velocity (or average speed), were obtained with each program. (A) ToxTrack interface window showing a tracking trace. (B) An interface from Animal Tracker, an ImageJ-based program. (C) An interface window from IdTracker, a MATLAB-based program. (D) Summary of results obtained in the anxiety behavioral tests. We tracked the video on a laptop computer Hewlett Packard, HP Pavilion x360 (processor Intel Core i3, 4 GB RAM, Windows 10, 64 bits).

illumination and contrast, which can affect the results depending on the error rate of each program (Appendix).

However, some of the softwares, such as Ctrax, Cowlog, and VideoHacking, do not need special video formats to work. Exported data, in some cases, can result in changes in the content (e.g., due to the misreading of decimal commas), so it is better to save the results in csv or txt files and then import them to the software used for data analysis or a spreadsheet.

As some programs are outdated and therefore do not work on some platforms, it is necessary to have updates for their adequate operation. For example, ZooTracer, another freely available software package developed by Microsoft Research in 2006, is available for download with the last update in 2014 (https://www.microsoft.com/en-us/research/project/ zootracer/). However, we did not include it in this review due to problems with the installation. On the other hand, it is important to mention that there are several options for the evaluation of different patterns of behavior and that some programs lack packages or updates for the wider analyses of behavior. Further developments are needed to create more precise and user-friendly free software for the automated analysis of complex and specific behaviors, such as fighting, courtship, and jumps, which might analyze the video by ROI with several animals simultaneously. In the videos analyzed, the resolution had to be downsampled on many occasions to avoid the software failing and closing unexpectedly. Another important limitation is the simultaneous processing capacity of two or more videos, which depends on the computer's RAM, the amount of time or frames processed, and the algorithms of each software. This would save much time, especially when a single subject was tested per video, and a considerable number of videos per experimental group were generated.

For the automated analysis of behavior, we consider it necessary that the minimum data analyzed include the following: distance, speed, freezing time, immobilization time, turn angle (customizable), and number of turns. The ROI should be adjustable to any geometric shape, including asymmetric shapes, and the number of entries, latency, and duration in an ROI should be analyzed (in addition to the variables mentioned above). The $X$ and $Y$ coordinates and the path graphs are important data for assessing behavior and simplifying the analysis process.

Due to the wide variety of analyses provided by each software package, we recommend using a combination of two, for example, ToxTrack or AnimalTracker plus JAABA or EthoWatcher. The first set can quantify the variables related to locomotion and the analysis of some social and cognitive behaviors, while the second set of programs can 

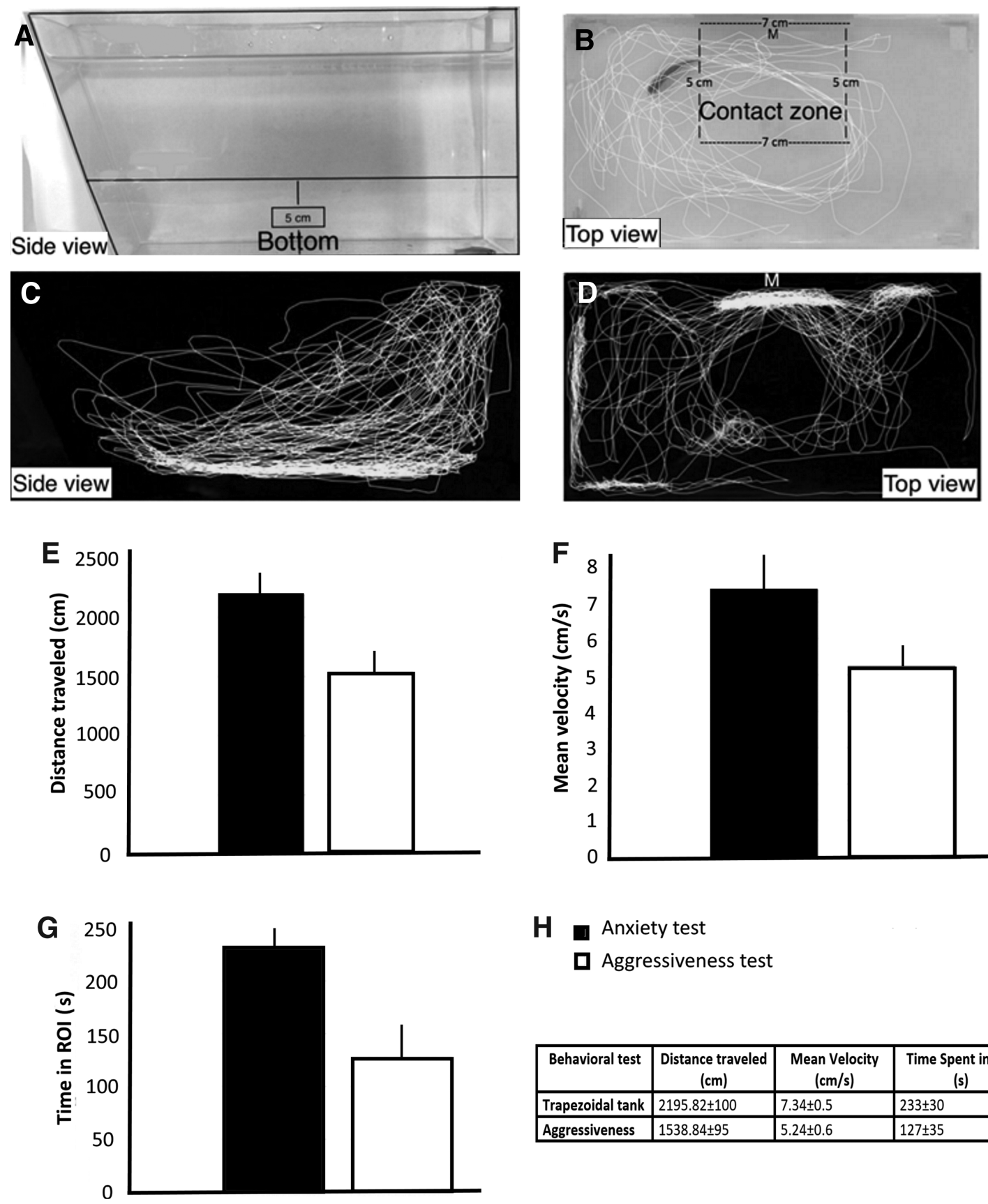

\begin{tabular}{|l|l|l|l|}
\hline Behavioral test & $\begin{array}{c}\text { Distance traveled } \\
(\mathbf{c m})\end{array}$ & $\begin{array}{c}\text { Mean Velocity } \\
(\mathrm{cm} / \mathbf{s})\end{array}$ & $\begin{array}{c}\text { Time Spent in ROI } \\
(\mathbf{s})\end{array}$ \\
\hline Trapezoidal tank & $2195.82 \pm 100$ & $7.34 \pm 0.5$ & $233 \pm 30$ \\
\hline Aggressiveness & $1538.84 \pm 95$ & $5.24 \pm 0.6$ & $127 \pm 35$ \\
\hline
\end{tabular}

FIG. 2. Animal tracker was used to analyze anxiety and aggressiveness in zebrafish. (A) Side view of the trapezoidal tank used to test anxiety behavior in zebrafish. Two areas were defined: bottom and top zones. (B) Top view of the rectangular tank used for the aggressiveness test with a lateral mirror $(\mathrm{M})$ with the contact area delimited by dotted lines. (C) Representative track of a zebrafish in the anxiety test obtained by AnimalTracker software. It was observed that zebrafish display high activity in the bottom area. (D) Representative track of a zebrafish in the aggressiveness test obtained by AnimalTracker software. In this test, zebrafish display high activity near the mirror. (E-H) Main results generated by AnimalTracker in the anxiety and aggressiveness behavioral tests. (E) Distance traveled (cm). (F) Mean velocity (cm/s). (G) Time spent in the ROI (s). (H) Summary of results obtained in the anxiety and aggressiveness behavior tests. Input data were obtained from the experiments shown in Figure 1. Error bars are \pm 1 SD. ROI, region of interest; SD, standard deviation.

quantify specific behaviors. Finally, something important to include in future reviews will be to contrast the results of the analysis of a single video in different programs, to compare automated analysis to manual analysis, and for some behaviors, to analyze locomotion (and social and cognitive tests) with several individuals with overlapping trajectories.

We think that the information reported in this study could be useful to researchers interested in working in the neuroscience field, especially people interested in a low-cost animal 
model, such as zebrafish, in combination with free tools for a behavioral analysis that provide simple, quick, inexpensive or free of cost, and reliable behavior analysis.

\section{Acknowledgments}

D.A.F. has been supported by research grants from Colciencias, Colombia, and VCTI-UAN. Funding sources had no role in the study design, in the collection, analysis and interpretation of data, in the writing of the report, or in the decision to submit the article for publication.

\section{Disclosure Statement}

No competing financial interests exist.

\section{References}

1. Hajar R. Animal testing and medicine. Heart Views 2011; $12: 42$.

2. Phifer-Rixey M, Nachman MW. Insights into mammalian biology from the wild house mouse Mus musculus. eLife 2015;4:e05959.

3. Arunachalam M, Raja M, Vijayakumar C, Malaiammal P, Mayden RL. Natural history of zebrafish (Danio rerio) in India. Zebrafish 2013;10:1-14.

4. Howe K, Clark MD, Torroja CF, Torrance J, Berthelot C, Muffato $\mathrm{M}$, et al. The zebrafish reference genome sequence and its relationship to the human genome. Nature 2013:496: 498-503.

5. Mouse Sequencing Consortium 2002. Initial sequencing and comparative analysis of the mouse genome. Nature 2002;420:520-562.

6. Adams MD, Celniker SE, Holt RA, Evans CA, Gocayne JD, Amanatides PG, et al. The genome sequence of Drosophila melanogaster. Science 2000;287:2185-2195.

7. Miczek K, Takahashi A, Gobrogge K, Hwa L, de Almeida R. Escalated aggression in animal models: shedding new light on mesocorticolimbic circuits. Curr Opin Behav Sci 2015;3:90-95.

8. Kalueff AV, Gebhardt M, Stewart AM, Cachat JM, Brimmer M, Chawla JS, et al. Towards a comprehensive catalog of zebrafish behavior 1.0 and beyond. Zebrafish 2013;10:70-86.

9. Bartholomew NR, Burdett JM, VandenBrooks JM, Quinlan MC, Call GB. Impaired climbing and flight behaviour in Drosophila melanogaster following carbon dioxide anaesthesia. Sci Rep 2015;5:15298.

10. Leger M, Quiedeville A, Bouet V, Haelewyn B, Boulouard $\mathrm{M}$, Schumann-Bard $\mathrm{P}$, et al. Object recognition test in mice. Nat Protoc 2013;8:2531-2537.

11. Peng M, Zhang C, Dong Y, Zhang Y, Nakazawa H, Kaneki $\mathrm{M}$, et al. Battery of behavioral tests in mice to study postoperative delirium. Sci Rep 2016;6:29874.

12. Piato AL, Capiotti KM, Tamborski AR, Oses JP, Barcellos LJ, Bogo MR, et al. Unpredictable chronic stress model in zebrafish (Danio rerio): behavioral and physiological responses. Prog Neuropsychopharmacol Biol Psychiatry 2011;35:561-567.

13. Budick SA, O'Malley DM. Locomotor repertoire of the larval zebrafish: swimming, turning and prey capture. J Exp Biol 2000;203:2565-2579.

14. Fero K, Yokogawa T, Burgess HA: The behavioral repertoire of larval zebrafish. In: Zebrafish models in neurobehavioral research. Kalueff A and Cachat J, (eds), pp. 249-291. Humana Press, Totowa, NJ, 2011.
15. Dankert H, Wang L, Hoopfer ED, Anderson DJ, Perona P. Automated monitoring and analysis of social behavior in Drosophila. Nat Methods 2009;6:297-303.

16. Luyten L, Schroyens N, Hermans D, Beckers T. Parameter optimization for automated behavior assessment: plugand-play or trial-and-error? Front Behav Neurosci 2014; $8: 28$.

17. Nussbaum-Krammer CI, Neto MF, Brielmann RM, Pedersen JS, Morimoto RI. Investigating the spreading and toxicity of prion-like proteins using the metazoan model organism C. elegans. J Vis Exp 2015;52321.

18. Selvaraj V, Santhakumar K. Analyzing locomotor activity in Zebrafish larvae using wrMTrck. Zebrafish 2017;14: 287-291.

19. Nema S, Hasan W, Bhargava A, Bhargava Y. A novel method for automated tracking and quantification of adult zebrafish behaviour during anxiety. J Neurosci Methods 2016;271:65-75.

20. Tungtur SK, Nishimune $N$, Radel J, Nishimune $H$. Mouse behavior tracker: an economical method for tracking behavior in home cages. Biotechniques 2017;63:215220.

21. Gulyas M, Bencsik N, Pusztai S, Liliom H, Schlett K. AnimalTracker: an ImageJ-based tracking API to create a customized behaviour analyser program. Neuroinformatics 2016:14:479-481.

22. Perez-Escudero A, Vicente-Page J, Hinz RC, Arganda S, de Polavieja GG. idTracker: tracking individuals in a group by automatic identification of unmarked animals. Nat Methods 2014;11:743-748.

23. Tort AB, Neto WP, Amaral OB, Kazlauckas V, Souza DO, Lara DR. A simple webcam-based approach for the measurement of rodent locomotion and other behavioural parameters. J Neurosci Methods 2016;157:91-97.

24. Kabra M, Robie AA, Rivera-Alba M, Branson S, Branson K. JAABA: interactive machine learning for automatic annotation of animal behavior. Nat Methods 2013;10:64-67.

25. Branson K, Robie AA, Bender J, Perona P, Dickinson MH. High-throughput ethomics in large groups of Drosophila. Nat Methods 2009;6:451-457.

26. Conklin EE, Lee KL, Schlabach SA, Woods IG. VideoHacking: Automated tracking and quantification of locomotor behavior with open source software. Journal of Undergraduate Neuroscience Education 2015;13:A120A125.

27. Rodriguez A, Zhang H, Klaminder J, Brodin T, Andersson PL, Andersson M. ToxTrac: a fast and robust software for tracking organisms. Methods Ecol Evol 2017;1-5.

28. Rodriguez A, Zhang H, Klaminder J, Brodin T, Andersson M. ToxId: an algorithm to track the identity of multiple animals. Sci Rep 2017;7:14774.

29. Crispim Junior CF, Pederiva CN, Bose RC, Garcia VA, Lino-de-Oliveira C, Marino-Neto J. ETHOWATCHER: validation of a tool for behavioral and video-tracking analysis in laboratory animals. Comput Biol Med 2012;42: 257-264.

30. Samson A, Ju L, Kim H, Zhang S, Lee J, Sturgeon S, et al. MouseMove: an open source program for semi-automated analysis of movement and cognitive testing in rodents. Sci Rep 2015;5:16171.

31. Hanninen L, Pastell M. CowLog: open-source software for coding behaviors from digital video. Behav Res Methods 2009;41:472-476. 
32. Schneider CA, Rasband WS, Eliceiri KW. NIH Image to ImageJ: 25 years of image analysis. Nat Methods 2012;9: 671-675.

33. Cachat J, Stewart A, Utterback E, Hart P, Gaikwad $\mathrm{S}$, Wong $\mathrm{K}$, et al. Three-dimensional neurophenotyping of adult zebrafish behavior. PLoS One 2011;6: e17597.

34. Mora-Zamorano FX, Svoboda KR, Carvan MJ. The nicotine-evoked locomotor response: A behavioral paradigm for toxicity screening in Zebrafish (Danio rerio) embryos and eleutheroembryos exposed to methylmercury. PLoS One 2016;11:e0154570.
Address correspondence to:

Rafael A. Vargas, $M D, P h D$

PhD Program in Health Sciences

School of Medicine

Universidad Antonio Nariño

Kr 3 Este \# 47 A - 15

Bogotá 110231

Colombia

E-mail: rvargas3200@hotmail.com antonio.vargas@uan.edu.co

\section{Appendix}

\section{Some Recommendations to Obtain High-Quality Video Recording}

After testing multiple freely available software packages, we have some suggestions based on our experience. These recommendations could be useful for students and researchers interested in the analysis of adult zebrafish behavior. Considering the following parameters will permit one to obtain high-quality videos and guarantee standardization in video recording.

Illumination is a key factor because it is one of the main factors that generates false recognition in the tracking of animals. Reflections and shadows should be reduced in the field before starting to record videos. To solve this problem, it is important to maintain the same lighting conditions throughout all recordings. The generation of a contrast between the field and the animals is the main objective; therefore, if the animal to be evaluated is dark, the field should be white, and vice versa. If shadows (or artifacts) are generated in the field, the lighting must be improved with LED lights and/or light reflectors. Some software packages have a size filter to track only the animal in the field, even if there are artifacts.

The recording camera used can vary from a simple webcam to a professional camera. In our experience, to maximize accuracy, we recommend a camera that records at least 30 frames per second (FPS) and has a resolution of $840 \times 420$ pixels. The accuracy in speed calculations may change because in most algorithms, the position (pixels at $X$ and $Y$ coordinates) is determined in each frame of the video, and therefore, higher resolution and FPS improve the accuracy of the obtained results. However, lower FPS and resolution can produce similar results if there is good contrast (between the field and the animal). It is important to note that some programs allow the video analysis with a resolution of $320 \times 240$ pixels, and this is allowed mostly with black and white video due to increases in speed and contrast.

The Audio Video Interleaved format with an MPEG or MJPEG compression is the most common format used by the software tested. There are free programs to convert video from one format to another format, and we recommend Avidemux (Mean.io ${ }^{\circledR}$; Linnovate, Bnei Brak, Israel) that allows the conversion of formats, changes in compression, size, color, and contrast, and the removal of unwanted regions of the field, among many other utilities. We recommend saving memory and processing time by converting or recording videos in grayscale.

Finally, it is necessary to stabilize the camera with a tripod, maintain the same distance between the lens of the camera and the field during all behavioral tests, and have measurement reference points, such as the length of the field or a ruler outside the arena. 